\title{
Reversible Restrictive Lung Disease in Pseudomesotheliomatous Carcinoma in a Lung Harboring a HER2-mutation
}

\author{
Shinya Sakata, Yasumiko Sakamoto, Akira Takaki, Shiho Ishizuka, \\ Sho Saeki and Kazuhiko Fujii
}

\begin{abstract}
:
Pseudomesotheliomatous carcinoma of the lung is very rare, and reversible restrictive lung disease with pseudomesotheliomatous carcinoma has not yet been previously reported. We herein report a patient with HER2-positive non-small-cell lung cancer (NSCLC) showing pseudomesotheliomatous carcinoma who was successfully treated with bevacizumab combination chemotherapy. A 56-year-old Japanese woman with advanced NSCLC presented with dyspnea. We administered chemotherapy with cisplatin $\left(75 \mathrm{mg} / \mathrm{m}^{2}\right)$ plus pemetrexed $\left(500 \mathrm{mg} / \mathrm{m}^{2}\right)$ plus bevacizumab $(15 \mathrm{mg} / \mathrm{kg})$, followed by pemetrexed plus bevacizumab. After eight cycles of maintenance chemotherapy, chest CT demonstrated a marked tumor reduction and an improvement of the right lung volume. The vital capacity was thereafter found to have significantly increased according to pulmonary function tests.
\end{abstract}

Key words: HER2, lung cancer, pseudomesotheliomatous carcinoma, restrictive lung disease

(Intern Med 57: 2223-2226, 2018)

(DOI: 10.2169/internalmedicine.9612-17)

\section{Introduction}

Pseudomesotheliomatous carcinoma of the lung is very rare, with a reported incidence among all lung cancers of $0.46 \%$ (1). Reversible restrictive lung disease with pseudomesotheliomatous carcinoma in non-small cell lung cancer (NSCLC) patients harboring human epidermal growth factor receptor-2 (HER2)-mutation has not yet been previously reported. Because of its rarity and clinical features, there are fewer evidence-based therapies available for pseudomesotheliomatous carcinoma of the lung compared to other types of NSCLC. A novel, effective treatment for NSCLC with pseudomesotheliomatous carcinoma is therefore needed.

We herein describe a patient with HER2-positive lung cancer showing pseudomesotheliomatous carcinoma who was successfully treated with bevacizumab combination chemotherapy and thereafter demonstrated a significant improvement in the symptoms of restrictive lung disease.

\section{Case Report}

A 56-year-old Japanese woman presented with dyspnea. She was not occupationally exposed to asbestos and had never smoked. Chest radiography and computed tomography (CT) revealed diffuse pleural thickening and a reduced volume of the right lung and narrowing of the intercostal spaces (Fig. 1A, C and D). A transbronchial lung biopsy of the mass was performed; the pathological diagnosis was adenocarcinoma. ${ }^{18}$ F-fluorodeoxyglucose (FDG) positronemission tomography (PET) demonstrated FDG accumulation in an area of diffuse pleural thickening (Fig. 1B). Molecular testing with next-generation sequencing revealed a HER 2 mutation in exon 20 insertion. We administered chemotherapy with cisplatin $\left(75 \mathrm{mg} / \mathrm{m}^{2}\right)$ plus pemetrexed $(500$ $\left.\mathrm{mg} / \mathrm{m}^{2}\right)$ and bevacizumab $(15 \mathrm{mg} / \mathrm{kg})$ every 3 weeks, followed by pemetrexed plus bevacizumab. After eight cycles of maintenance chemotherapy, CT and PET demonstrated a marked tumor reduction and an improvement of diffuse 

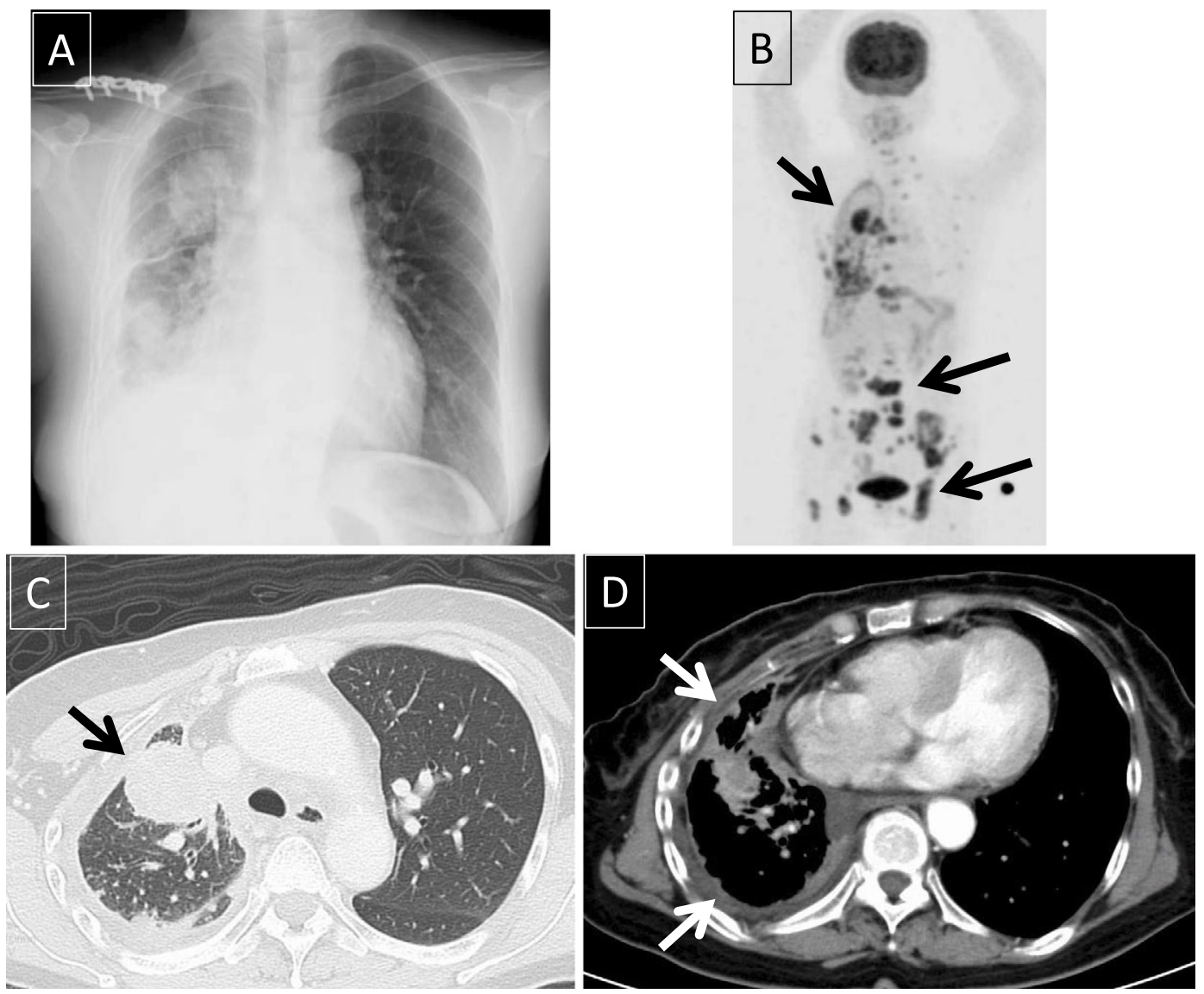

Figure 1. A: Chest radiograpy showing a mass with diffuse pleural thickening and a reduced volume of the right lung. B: PET-CT demonstrating FDG accumulation in the mass and diffuse pleural thickening and multiple bones before chemotherapy (arrow). C, D: Chest CT demonstrating a mass and diffuse pleural thickening and reduced volume of the right lung with narrowing of the intercostal spaces (arrow).

pleural thickening and the right lung volume (Fig. 2). The vital capacity and peak expiratory flow then was observed to significantly increase according to pulmonary function tests (Fig. 3).

\section{Discussion}

Reversible restrictive lung disease of lung cancer patients is generally observed in cases with malignant pleural effusion or obstructive atelectasis due to the presence of a tumor. However, reversible restrictive lung disease with pseudomesotheliomatous carcinoma as observed in our patient has not yet been reported. Pseudomesotheliomatous carcinoma was first reported by Harwood et al. in 1976, among lung cancer patients with extension to the pleura (2). Pseudomesotheliomatous carcinoma of the lung is very rare, with a reported incidence among all lung cancers of $0.46 \%$. It was reported that $87 \%$ of pseudomesotheliomatous carcinoma patients had a smoking history and $76 \%$ of such cases had an asbestos exposure history (3). Smoking and asbestos have thus been considered as causes of pseudomesotheliomatous carcinoma carcinogenesis.

A case of pseudomesotheliomatous carcinoma harboring an epidermal growth factor receptor (EGFR) mutation without occupational asbestos exposure was recently reported, with narrowing of the intercostal spaces (4). Although previous pseudomesotheliomatous carcinoma reports did not describe the incidence of oncogenes, such as EGFR mutation or HER2 mutation, the present case might indicate that the HER 2 mutation could play an important role in the development of pseudomesotheliomatous carcinoma of the lung, as our patient had no other risk factors (e.g., smoking history, asbestos exposure).

HER2 mutation is a rare somatic mutation that represents 2-3\% of lung cancers, and there has been no report of its comorbidity with pseudomesotheliomatous carcinoma showing intercostal space narrowing $(5,6)$. Regarding the outcomes of chemotherapy for advanced HER2-mutant lung cancers, Mazieres et al. reported the outcomes with conventional chemotherapy excluding HER2 targeted drugs. The response rate and the median progression free survival for patients receiving first-line chemotherapy were $43.5 \%$ and 6.0 months [95\% confidence interval (CI): 5.0-7.1], respectively $(n=93)$ (7). In addition, Falchook et al. reported a case of advanced non-small cell lung cancer harboring a HER $2 \mathrm{mu}-$ tation which achieved a sustained antitumor effect to la- 

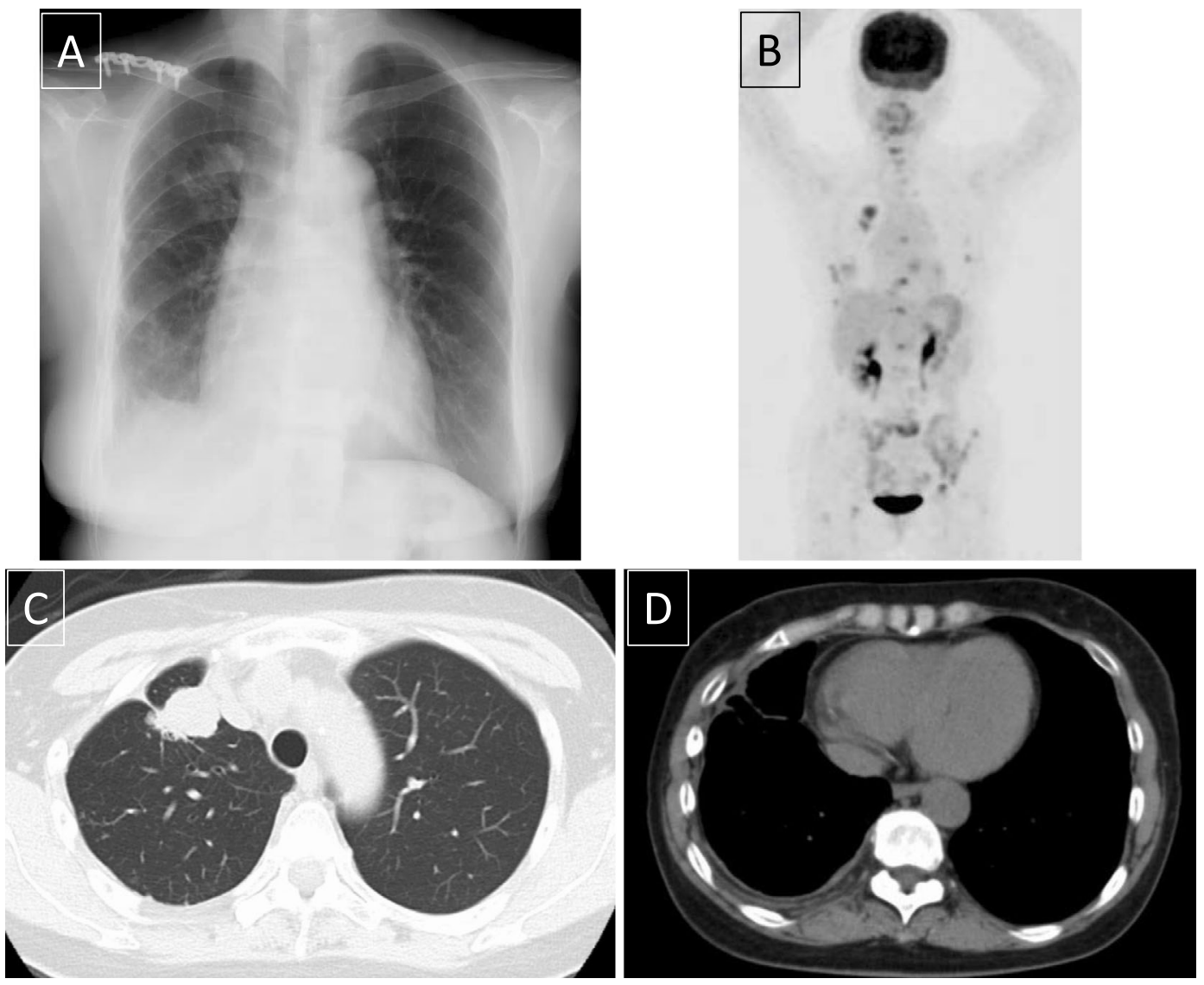

Figure 2. Chest radiograpy and CT demonstrating a marked tumor reduction and an improvement of diffuse pleural thickening and the right lung volume after eight cycles of maintenance chemotherapy.
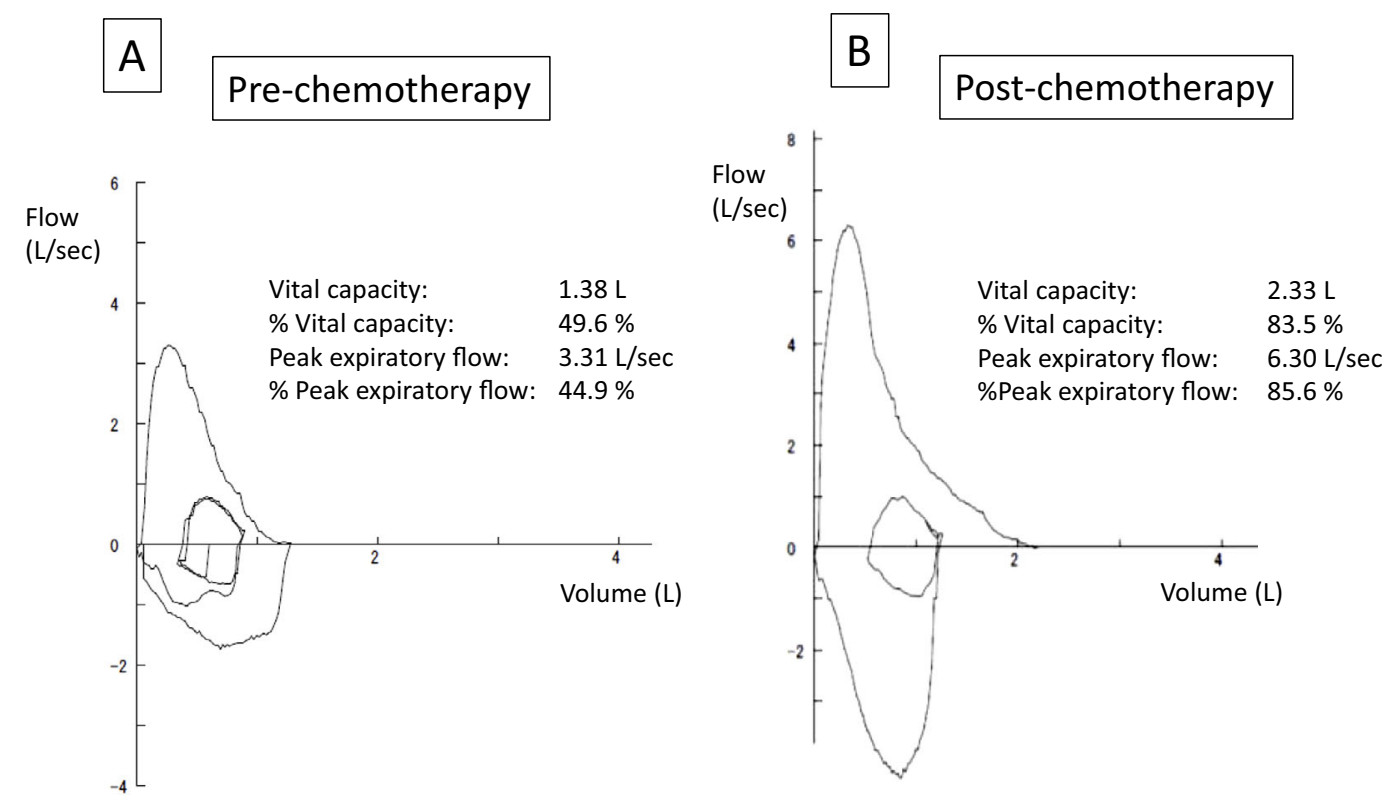

Figure 3. Pulmonary function tests showing a significant improvement in the vital capacity and peak expiratory flow.

patinib and trastuzumab in combination with bevacizumab (8). In the present case, we performed bevacizumab combination chemotherapy. The findings of this case suggest that bevacizumab containing chemotherapy may play an important role in the successful treatment of pseudomesotheliomatous carcinoma of the lung harboring a HER2- 
mutation.

Regarding prognostic factors, Tomizawa et al. reported that HER2 mutations were not associated with the prognosis of non-small-cell lung cancer patients, suggesting a negative prognostic role for the co-existence of a TP53 mutation (9).

The present report is the first to described a case of HER2-positive lung cancer showing pseudomesotheliomatous carcinoma that was successfully treated with cisplatin plus pemetrexed and bevacizumab chemotherapy with a significant improvement in the symptoms of restrictive lung disease.

The authors state that they have no Conflict of Interest (COI).

\section{References}

1. Kobashi Y, Matsushita T, Irei T. Clinicopathological analysis of lung cancer resembling malignant pleural mesothelioma. Respirology 10: 660-665, 2005.

2. Harwood TR, Gracey DR, Yokoo H. Pseudomesotheliomatous carcinoma of the lung. A variant of peripheral lung cancer. Am J Clin Pathol 65: 159-167, 1976.

3. Attanoos RL, Gibbs AR. 'Pseudomesotheliomatous' carcinomas of the pleura: A 10-year analysis of cases from the Environmental Lung Disease Research Group, Cardiff. Histopathology 43: 444-
452, 2003.

4. Takahara Y, Nishiki K, Nakase K, et al. A case of diffuse pleural invasion of pseudomesotheliomatous carcinoma with EGFR mutation diagnosed by thoracoscopic biopsy. J Jpn Soc Respir Endoscopy 38: 183-189, 2016.

5. Stephens $P$, Hunter C, Bignell G, et al. Lung cancer: intragenic ERBB2 kinase mutations in tumours. Nature 431: 525-526, 2004.

6. Arcila ME, Chaft JE, Nafa K, et al. Prevalence, clinicopathologic associations, and molecular spectrum of ERBB2 (HER2) tyrosine kinase mutations in lung adenocarcinomas. Clin Cancer Res 18: 4910-4918, 2012.

7. Mazieres J, Barlesi F, Fileron T, et al. Lung cancer patients with HER2 mutations treated with chemotherapy and HER2-targeted drugs: results from the European EUHER2 cohort. Ann Oncol 27: 281-286, 2016.

8. Falchook GS, Janku F, Tsao AS, Bastida CC, Stewart DJ, Kurzrock R. Non-small-cell lung cancer with HER2 exon 20 mutation: regression with dual HER2 inhibition and anti-VEGF combination treatment. J Thorac Oncol 8: e19-e20, 2013.

9. Tomizawa K, Suda K, Onozato R, et al. Prognostic and predictive implications of HER2/ERBB2/neu gene mutations in lung cancers. Lung Cancer 74: 139-144, 2011.

The Internal Medicine is an Open Access article distributed under the Creative Commons Attribution-NonCommercial-NoDerivatives 4.0 International License. To view the details of this license, please visit (https://creativecommons.org/licenses/ by-nc-nd/4.0/).

(C) 2018 The Japanese Society of Internal Medicine

Intern Med 57: 2223-2226, 2018 